\title{
DETERMINAÇÃO DO CONFORTO TÉRMICO DE VACAS LEITEIRAS UTILIZANDO A MINERAÇÃO DE DADOS \\ EVALUATION OF THERMAL COMFORT IN DAIRY CATTLE USING DATA MINING
}

\author{
MAURÍCIO PERISSINOTTO' \\ DANIELLA J. DE MOURA ${ }^{2}$
}

\section{RESUMO}

Vários estudos já demostraram que o desempenho animal é influenciado pelo ambiente térmico e que a intensidade com que isso ocorre é função da região e dos animais com que se está trabalhando, o que fez surgir vários relatos sobre os limites do Índice de Temperatura e Umidade (ITU). Sendo assim, o objetivo deste estudo foi modelar e avaliar o impacto do estresse térmico pelo uso da Mineração de Dados meteorológicos (temperatura e umidade relativa do ar) e de um conjunto de classes oriundas da integração da base de regras dos dados fisiológicos (temperatura retal e freqüência respiratória) e assim determinar os intervalos críticos do ITU. O banco de dados foi formado no município de São Pedro - SP. Para isso foi utilizado o programa computacional WEKA ${ }^{\circledR}$, versão 34, que executou tarefa de classificação, utilizando o algoritmo J48 em validação cruzada para a construção da árvore de decisão. Os resultados obtidos por intermédio da Mineração de Dados meteorológicos e fisiológicos permitiram adaptar o ITU em função da região e dos animais utilizados. A Mineração de Dados também permitiu estabelecer alguns parâmetros ideais de conforto aos animais para as condições de estudo. Observou-se que valores do ITU por volta de 77 proporcionam condição de conforto para os animais confinados e quando o ITU atinge valores acima de 80 a situação é de estresse térmico alto.

Palavras-Chaves: produção animal, estresse térmico, índice de temperatura e umidade.

1.Engenheiro Agrônomo, MSc, Departamento de Engenharia Rural, ESALQ /USP, Piracicaba mperissi@esalq.usp.br

2.Engenheira Agrônoma. Prof. Dr. FEAGRI-UNICAMP. Campinas, SP. 


\section{ABSTRACT}

Some studies had already had shown that the animal performance is influenced by the thermal environment and that the intensity that it occurs is function of the region that the animals are lodged, questioning the limits of the temperature and humidity index (THI). The objective of this study was to model and to evaluate the impact of the thermal stress using the Data Mining of meteorological data (air temperature and relative humidity) and physiological data (rectal temperature and respiratory rate) and thus to determine the critical intervals of the THI. The data base was built with meteorological and physiological data from a commercial farm located in the city of São Pedro - SP. For the Data Mining process the computational program Weka was used, version 3-4, using the J48 algorithm to build a decision tree. According to the results it is possible to adapt the THI in function of the region and the animals using the Data Mining of meteorological and physiological data. The analysis of the data base indicated a high capacity of the model in representing the classes of comfort from this data set. It was found that when the internal THI is lower or equal the 77 the thermal comfort is high, and when it is higher than 80 is low.

Keywords: animal production, thermal stress, temperature and humidity index.

\section{INTRODUÇÃO}

O ambiente térmico exerce forte influência sobre o desempenho animal, uma vez que afeta os mecanismos de transferência de calor e, portanto, a regulação do balanço térmico entre o animal e o meio. Para caracterizar ou quantificar as zonas de conforto térmico adequadas às diferentes espécies animais foram desenvolvidos índices de conforto térmico (entre eles o ITU), que conjugam em uma única variável os elementos que caracterizam o ambiente térmico associado ao animal e as suas influências no conforto deste. Este índice de conforto é utilizado para estabelecer classes que são definidas de acordo com a reação fisiológica e/ou comportamental do animal.

O animal dentro de uma faixa de ITU considerada adequada produzirá de acordo com o seu potencial genético. Existem vários relatos sobre os limites do ITU, porém, é preciso levar-se em consideração que estes limites podem sofrer variações em função da região e dos animais utilizados na propriedade e, portanto, fazem-se necessárias adaptações do índice de conforto térmico para que ele possa ser convenientemente utilizado em uma região diferente daquela onde foi desenvolvido.

A capacidade do animal de resistir às condições de estresse calórico tem sido avaliada fisiologicamente por alterações na temperatura retal e freqüência respiratória. Através de bancos de dados fisiológicos e de estações meteorológicas há a possibilidade de modelagem e avaliação do impacto do estresse térmico pelo uso da Mineração de Dados. A Mineração de Dados é definida como processo de extração de conhecimento a partir de bancos de dados, objetivando utilizar este conhecimento adquirido em um processo decisório (REZENDE et al., 2003). 
O objetivo deste estudo foi modelar e avaliar o impacto do estresse térmico pelo uso da Mineração de Dados meteorológicos e de um conjunto de classes oriundas da integração da base de regras dos dados fisiológicos, determinando os intervalos críticos do Índice de Temperatura e o objetivo deste estudo foi modelar e avaliar o impacto do estresse térmico pelo uso da Mineração de Dados meteorológicos e de um conjunto de classes oriundas da integração da base de regras dos dados fisiológicos, determinando os intervalos críticos do Índice de Temperatura e Umidade (ITU).

\section{MATERIAIS E MÉTODOS}

O estudo foi desenvolvido em uma fazenda de produção leiteira comercial, localizada no município de São Pedro situada no interior do Estado de São Paulo, latitude $22^{\circ} 32^{\prime} 55^{\prime \prime}$ Sul e longitude $47^{\circ}$ 54' $50 "$ Oeste, estando a cerca de $580 \mathrm{~m}$ de altitude. Tem um clima CWA da classificação Koppen, ou seja, quente e úmido, com estação chuvosa no verão e seco no inverno.

Para a análise física do ambiente interior da instalação, sistemas de aquisição de dados $\mathrm{HOBO}^{\circledR}$ foram dispostos no centro geométrico da instalação, a aproximadamente $3 \mathrm{~m}$ de altura. Estes dataloggers registraram os valores da temperatura de bulbo seco e da umidade relativa do ar a cada 15 min ao longo das $24 \mathrm{~h}$ durante 0 período de coleta de dados, possibilitando o cálculo do Índice de Temperatura e Umidade (ITU) de acordo com a equação desenvolvida originalmente por THOM (1959): ITU = Tbs + 0,36 Tpo + 41,2, em que Tbs é a temperatura de bulbo seco $\left({ }^{\circ} \mathrm{C}\right)$ e Tpo é a temperatura do ponto de orvalho $\left({ }^{\circ} \mathrm{C}\right)$.
Para a climatização da instalação, utilizaram-se ventiladores na área de alimentação dos animais e na área de descanso associados a um sistema de aspersão montado logo abaixo dos ventiladores, sobre o cocho de alimentação dos animais, constituídos de bicos com vazão de 30 L.hora $^{-1}$ por bico e com intermitência de 12 min (1 min ligado e 11 min desligado). O sistema de climatização teve o funcionamento controlado por um termostato e um timer e era acionado quando a temperatura ambiente ultrapassava $24^{\circ} \mathrm{C}$.

Para a coleta dos dados fisiológicos foram utilizadas 15 vacas holandesas, multíparas, em lactação, com peso médio de $650 \mathrm{~kg}$. Os animais foram selecionados de acordo com os dias de lactação (média de 180 dias) e produção de leite e divididos em 3 grupos (produção menor que $20 \mathrm{~L}$, de 20 a $25 \mathrm{~L}$ e maior que $25 \mathrm{~L}_{\text {animal }}{ }^{-1} \mathrm{dia}^{-1}$ ). Os animais foram mantidos em abrigo do tipo freestall e permaneceram durante todo o dia com livre acesso às baias e demais áreas do abrigo, mas sem acesso à pastagem. Os dados fisiológicos monitorados foram temperatura retal (termômetro clínico digital inserido no reto) e freqüência respiratória (contagem dos movimentos da região do flanco durante $15 \mathrm{~s}$ ). A coleta dos dados fisiológicos foi a freqüência respiratória (contagem dos movimentos da região do flanco durante $15 \mathrm{~s})$. A coleta dos dados fisiológicos foi feita durante nove dias não consecutivos do mês de Novembro de 2003, realizadas às 09h:00min, 11h:00min, 13h:00min, 15h:00 min e $17 \mathrm{~h}: 00 \mathrm{~min}$.

Os animais receberam água e alimento à vontade durante todo o período de experimento e foram realizadas 3 ordenhas diárias (03h:00min, 10h:00min e 17h:00min), sendo os dados de produção de leite registrados de acordo com o 
controle da fazenda.

No processo de Mineração de Dados o programa computacional utilizado para as análises foi o Waikato Environment for Knowledge Analysis (WEKA ${ }^{\circledR}$ ), versão 34, que executou tarefa de classificação, utilizando o algoritmo J48 em validação cruzada (cross validation) para a construção da árvore de decisão. A validação cruzada dividiu o conjunto de dados em dez partes de igual tamanho, sendo nove utilizadas para o treinamento e uma utilizada para o teste. Sendo assim, a classificação foi repetida dez vezes e a parte do teste foi usada para validar a parte de treinamento, melhorando as regras geradas para as classes (REZENDE et al., 2003).

O processo de Mineração de Dados foi dividido em fases, restringindo um conjunto de ações definidas para cada uma delas, desencadeadas de forma cíclica, de acordo com a metodologia CRISP-DM (CHAPMAN et al., 2000).

Foi feita a transformação dos valores numéricos da temperatura retal (TR) e da freqüência respiratória $(F R)$ em escalas simbólicas, que foram posteriormente relacionadas para a caracterização lingüística da sensação de conforto térmico dos animais. Segundo a recomendação de diversos trabalhos da literatura (MCDOWELL et al., 1954; KOLB, 1987;
IGONO e JOHNSON, 1990; STOBER, 1993; MARTELLO, 2002; PERISSINOTTO, 2003; MATARAZZO, 2004) os dados de temperatura retal foram divididos da seguinte forma: se TR $£ 38,8{ }^{\circ} \mathrm{C}$ então conforto alto; se $38,8{ }^{\circ} \mathrm{C}>$ TR $£ 39,2^{\circ} \mathrm{C}$ então conforto médio; se TR $>39,2{ }^{\circ} \mathrm{C}$ então conforto baixo. Já para a freqüência respiratória, segundo a literatura (BERMAN et al., 1985; BACCARI JÚNIOR et al., 1995; HAHN et al., 1997;STOWELL, 2000; NÄÄS e ARCARO JÚNIOR, 2001;MARTELLO, 2002; PERISSINOTTO,2003; MATARAZZO, 2004, MARTELLO, 2006), os dados foram considerados da seguinte forma: se FR $£$ 56 mov min ${ }^{-1}$ então conforto alto; se 56 mov $\mathrm{min}^{-1}>\mathrm{FR} £ 64 \mathrm{mov}^{\mathrm{min}^{-1}}$ então conforto médio; se FR > 64 mov min $^{-1}$ então conforto baixo.

O atributo meta foi estipulado em função da relação entre os limites da temperatura retal e da freqüência respiratória recomendados pela literatura, sendo feita a caracterização linguística da sensação de conforto dos animais para a entrada no modelo em: muito bom, bom, regular e ruim. As classificações são apresentadas na Tabela 1 .

O banco de dados para análise foi formado em uma única planilha pelos dados meteorológicos e pelas classes oriundas da integração da base de regras dos dados

TABELA 1 - Caracterização linguística da sensação de conforto térmico como função da temperatura retal $\left({ }^{\circ} \mathrm{C}\right)$ e da freqüência respiratória $\left(\mathrm{mov} \mathrm{min}^{-1}\right)$

\begin{tabular}{lccc}
\hline \multirow{2}{*}{$\begin{array}{c}\text { Temperatura retal } \\
\left({ }^{\circ} \mathrm{C}\right)\end{array}$} & \multicolumn{3}{c}{ Freqüência respiratória $\left(\right.$ mov.min $\left.^{-1}\right)$} \\
\cline { 2 - 4 } & $\begin{array}{c}\text { Conforto alto } \\
(\leq 56)\end{array}$ & $\begin{array}{c}\text { Conforto médio } \\
(56-64)\end{array}$ & $\begin{array}{c}\text { Conforto baixo } \\
(>64)\end{array}$ \\
\hline Conforto alto $(\leq 38,8)$ & Muito bom & Bom & Regular \\
Conforto médio $(38,8-39,2)$ & Bom & Regular & Ruim \\
Conforto baixo $(>39,2)$ & Regular & Ruim & Ruim \\
\hline
\end{tabular}


fisiológicos sendo que, cada linha correspondia à informação de um determinado dia e horário da estação meteorológica (temperatura de bulbo seco e umidade relativa), ao ITU calculado e as classes da caracterização linguística da sensação de conforto dos animais em função das observações fisiológicas (temperatura retal e frequência respiratória).

Com o objetivo de observar se há variação no modelo devido a diferença no nível produtivo dos animais, os dados foram analisados de duas maneiras: uma com a base de dados total dorebanho, e outra com a separação por produção, de acordo com três grupos de produção de leite (produção menor que $20 \mathrm{~L}$, de 20 a $25 \mathrm{~L}$ e maior que $25 \mathrm{~L}$. animal $\left.^{-1} \mathrm{dia}^{-1}\right)$.

Uma vez que o modelo de classificação foi construído, um conjunto de regras foi obtido lendo a árvore, de cima para baixo, até alcançar cada nó folha. Como resultado final, alcançou-se um conjunto ordenado de regras. Cada caminho possível da árvore correspondeu a uma regra do tipo "SE <condicional> ENTÃO $<$ conseqüente>".

A avaliação do modelo baseou-se em critérios de precisão, compreensibilidade e grau de interesse. $O$ conhecimento extraído foi interpretado de acordo com as exigências do sistema de produção e comparado ao conhecimento pré-existente, para verificação de conflitos, de conformidades, e para verificar se a informação descoberta era nova e útil. A precisão geral do modelo gerado foi obtida pela análise dos dados classificados corretamente (Correctly Classified Instances). A taxa de acerto das hipóteses geradas para cada classe de conforto térmico estudada foi estimada utilizando-se os critérios de precisão (precision) e de acertos (recall).

\section{RESULTADOS E DISCUSSÃO}

Observou-se na análise dos dados que não houve diferença considerável nos valores críticos de ITU entre os algoritmos resultantes da base de dados para vacas em diferentes níveis de produção de leite.

Portanto, os resultados apresentados referem-se ao algoritmo resultante da base de dados do rebanho total.

O algoritmo testado apresentou medidas de desempenho relevantes à sua performance com o tipo de conjunto de dados estudados. A porcentagem de instâncias classificadas corretamente, que caracteriza a precisão do modelo, foi de $96,9 \%$; e a classificação incorreta foi de $3,1 \%$. A distribuição da acurácia (precision) por classe foi próximo de 1 e a matriz de confusão bastante consistente, como pode ser visto na parte do algoritmo da saída do processo de Mineração de Dados apresentada no Quadro 1.

A matriz de confusão é mais uma medida que confirma o bom desempenho do algoritmo, pois somente $3,1 \%$ das 578 instâncias estão fora da diagonal principal, indicando uma alta capacidade do modelo em representar as classes de conforto para este conjunto de dados.

A Figura 1 mostra os valores críticos de conforto alto (sem estresse), conforto médio (estresse moderado) e conforto baixo (estresse alto) do ITU, encontrados para vacas Holandesas em lactação, em função das variáveis fisiológicas: temperatura retal e freqüência respiratória. 
$===$ Summary $===$

$$
===\text { Stratified cross-validation }===
$$

Correctly Classified Instances $\quad 560 \quad 96.8858 \%$

Incorrectly Classified Instances $18 \quad 3.1142 \%$

Kappa statistic $\quad 0.9582$

Mean absolute error $\quad 0.0264$

Root mean squared error $\quad 0.1186$

Relative absolute error

$7.0718 \%$

Root relative squared error

Total Number of Instances

$27.4613 \%$

578

$===$ Detailed Accuracy By Class $===$

TP Rate FP Rate Precision Recall F-Measure Class

$\begin{array}{llllll}0.934 & 0.009 & 0.972 & 0.934 & 0.953 & \text { muito_bom } \\ 0.964 & 0.005 & 0.988 & 0.964 & 0.976 & \text { regular } \\ 1 & 0.002 & 0.991 & 1 & 0.996 & \text { ruim } \\ 0.986 & 0.025 & 0.929 & 0.986 & 0.957 & \text { bom }\end{array}$

$===$ Confusion Matrix $===$

a $\quad b \quad c \quad d \quad<$ classified as

\begin{tabular}{llll|l}
141 & 1 & 0 & 9 & a $=$ muito_bom
\end{tabular}

QUADRO 1 - Dados resultantes que formaram o algoritmo da saída do processo de Mineração de Dados.

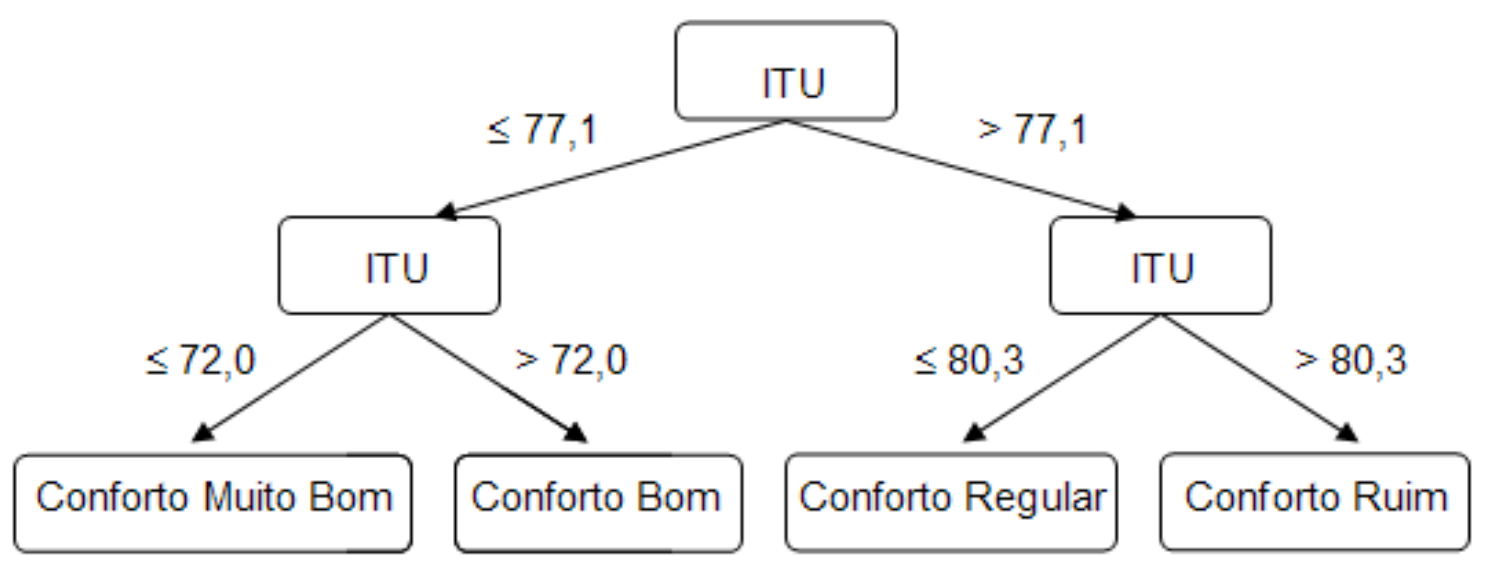

FIGURA 1 - Árvore de decisão para o Índice de Temperatura e Umidade 
As regras para a construção do modelo para as classes de conforto foram: Regra 1: SE ITU interno £ 72 ENTÃO o conforto térmico é MUITO BOM; Regra 2 SE ITU interno $72>$ ITU $£ 77,1$ ENTÃO conforto térmico é BOM; Regra 3 - SE 77,1 $>$ ITU interno £ 80,3 ENTÃO conforto térmico é REGULAR; Regra 4 - SE ITU interno > 80,3 ENTÃO conforto térmico é
RUIM.

Em função dos valores críticos do ITU encontrados na Figura 1, estabeleceram-se as classes do ITU em função da temperatura e da umidade relativa do ar para vacas Holandesas em lactação confinadas e para a região em estudo. Os resultados estão apresentados na Figura 2.

FIGURA 2 - Classes do Índice de Temperatura e Umidade

\begin{tabular}{|c|c|c|c|c|c|c|c|c|c|c|c|c|c|c|c|}
\hline UR & \multicolumn{15}{|c|}{ Temperatura de bulbo $\sec 0\left({ }^{\circ} \mathrm{C}\right)$} \\
\hline$(\%)$ & 22 & 23 & 24 & 25 & 26 & 27 & 28 & 29 & 30 & 31 & 32 & 33 & 34 & 35 & 36 \\
\hline 20 & (4) & 73 & 47 & 46. & (6) & 196. & (3) & Wh & 72,9 & 74,2 & 75,4 & 76,7 & 78,0 & 79,3 & 80,6 \\
\hline 30 & 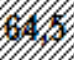 & 850 & 1) & 86 & 19. & Wa & 72,4 & 73,7 & 75,0 & 76,3 & 77,6 & 78,9 & 80,2 & 81,5 & 82,8 \\
\hline 40 & 6.8. & 61 & 6. & 10. & 3 & 72,6 & 73,9 & 75,3 & 76,6 & 77,9 & 79,2 & 80,5 & 81,9 & 83,2 & 84,5 \\
\hline 50 & 4 & 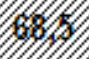 & 6 & 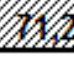 & 72,5 & 73,8 & 75,2 & 76,5 & 77,8 & 79,2 & 80,5 & 81,8 & 83,2 & 84,5 & 85,8 \\
\hline 60 & (6) & 45 & 1. & 72,2 & 73,5 & 74,9 & 76,2 & 77,6 & 78,9 & 80,2 & 81,6 & 82,9 & 84,2 & 85,6 & 86,9 \\
\hline 70 & 9 & 10 & 1 & 73,1 & 74,4 & 75,8 & 77,1 & 78,5 & 79,8 & 81,2 & 82,5 & & ifc & & Bom \\
\hline 80 & 69 & 1 & 72,5 & 73,9 & 75,2 & 76,6 & 77,9 & 79,3 & 80,6 & 82,0 & 83,3 & & onf & Bo & \\
\hline 90 & 79 & 41 & 73,2 & 74,6 & 75,9 & 77,3 & 78,6 & 80,0 & 81,3 & 82,7 & 84,1 & & onf & & \\
\hline 100 & 13 & 72,5 & 73,8 & 75,2 & 76,6 & 77,9 & 79,3 & 80,6 & 82,0 & 83,4 & 84,7 & & onfort & Ruim & \\
\hline
\end{tabular}

Os resultados encontrados neste trabalho diferem dos encontrados por alguns autores quanto ao ITU que reflete uma condição de conforto Bom ou Muito Bom para os animais. Os resultados encontrados sugerem que esta condição é atingida a um valor de ITU maior que o proposto por HAHN (1985), DU PREEZ et al. (1990) e IGONO \& JOHNSON (1990), que sugerem os valores de 70, 70 e 72, respectivamente. Da mesma forma, os valores de ITU que sugerem estresse térmico alto (conforto ruim) encontrados neste trabalho estão acima dos sugeridos pela literatura, sendo mencionados que esse estresse alto ocorre quando os valores de ITU estão acima de 79 (HAHN, 1985), acima de 78 (DU PREEZ et al., 1990) e acima de 76 (IGONO \& JOHNSON, 1990).

Por outro lado, os resultados encontrados estão de acordo com MARTELLO (2002), que não observou alteração na termorregulação de vacas Holandesas em condição de conforto, quando as médias diárias do ITU não ultrapassaram 76; e MATARAZZO (2004), que não verificou presença de estresse em vacas Holandesas quando analisou as variáveis temperatura retal e freqüência respiratória, mesmo sob ambiente com ITU acima de 75.

Na Figura 2, abaixo da temperatura de $22^{\circ} \mathrm{C}$, a sensação de conforto térmico é muito boa, independentemente dos valores de umidade relativa do ar. O limite superior da zona de termoneutralidade (ZTN) de vacas Holandesas em lactação situa-se próximo dos $26^{\circ} \mathrm{C}$ de temperatura de bulbo seco, independente da umidade relativa, diferindo do recomendado por BACCARI JUNIOR (1998), que recomenda que este limite seja de $24^{\circ} \mathrm{C}$.

Por outro lado, os resultados encontrados estão próximos ao sugerido por 
FUQUAY (1997), que considerou para o gado europeu valores de temperatura crítica superior entre 25 e $26^{\circ} \mathrm{C}$ para vacas em lactação, independente desta ter sido submetida à aclimatação prévia ou nível de produção. Já HUBER (1990), que considerou como adequadas para o conforto térmico de vacas em lactação temperatura do ar entre 4 e $26^{\circ} \mathrm{C}$.

É possível observar pela Figura 2 a importância da umidade relativa no conforto térmico, sendo que, quanto maior o valor dessa variável climática menor são os valores aceitáveis da temperatura do ar para que o animal continue em conforto. Esta afirmação também foi feita por PENNINGTON \& VANDEVENDER (2005), os quais observaram que sinais de moderado estresse térmico ocorrem quando a temperatura está entre 27 e $32^{\circ} \mathrm{C}$ com umidade maior que $50 \%$, e que em temperaturas entre 32 e $38^{\circ} \mathrm{C}$ e umidade maior que $50 \%$ as vacas mostram sinais evidentes de estresse térmico. Segundo os resultados apresentados, ambientes com temperatura acima de $34^{\circ} \mathrm{C}$ já podem ser considerados altamente estressantes, independentemente da umidade relativa do ar.

Os limites de conforto encontrados nesta pesquisa permitem afirmar que as vacas leiteiras no Brasil estão, em grande parte do ano e por várias horas por dia, sujeitas ao estresse térmico, pois, freqüentemente são registradas temperaturas superiores a estas.

\section{CONCLUSÕES}

De acordo com os resultados apresentados neste trabalho, pode-se afirmar que a temperatura retal e a freqüência respiratória apresentaram associação com o Índice de Temperatura e Umidade para vacas Holandesas em lac- tação submetidas a condição de confinamento.

A Mineração de Dados, além de auxiliar na tomada de decisão, pode ser utilizada na determinação de estratégias apropriadas, dando suporte ao produtor no gerenciamento de informações e no controle do processo de climatização das instalações.

A utilização da ferramenta permitiu estabelecer alguns parâmetros ideais de conforto aos bovinos leiteiros da raça holandesa em lactação, manejados em condição de confinamento total. Os resultados obtidos podem ser programados em um software que determine, com elevado grau de certeza, a ocorrência do estresse térmico em vacas em lactação, auxiliando na tomada de decisão do melhor momento para se acionar sistemas de climatização, de acordo com as condições climáticas de exposição, refletindo diretamente nas formas de manejo e custos de produção.

\section{REFERÊNCIAS BIBLIOGRÁFICAS}

BACCARI JUNIOR, F. Adaptação de sistemas de manejo na produção de leite em clima quente. In: SIMPÓSIO BRASILEIRO DE AMBIÊNCIA NA PRODUÇÃO DE LEITE, Piracicaba, 1998. Anais... Piracicaba: FEALQ, 1998. p.24 67.

BACCARI JUNIOR, F.; AGUIAR, I.S.; TEODORO, S. M. Hipertemia, taquipinéia e taquicardia em vacas Holandesas malhadas de vermelho sob estresse térmico. In: CONGRESSO BRASILEIRO DE BIOMETEOROLOGIA, 3., 1995, Jaboticabal. Anais... Jaboticabal: FUNEP, 1995. p. 15-16. 
BERMAN, A; FOLMAN, Y; KAIM, M.; MAMEN, M.; HERZ, Z; WOLFENSON, D. ARIELI, A.; GRABER, Y. Upper critical temperatures and forced ventilation effects for high-yielding dairy cows in a subtropical climate. Journal of Dairy Science, Savoy, IL v.68, n.6, jun., p. 1488-95, 1985.

CHAPMAN, P.; CLINTON, J.; KERBER, R.; KHABAZA, T.; REINARTZ, T.; SHEARER, C.; WIRTH, R. CRISP-DM 1.0. Step-by-step data mining guide. 78 p. 2000. Disponível em: <http://www.crisp-dm.org/CRISPWP0800.pdf>. Acesso em: 10 apr. 2007

DU PREEZ, J.D.; GIESECKE, W.H.; HATTINGH, P.J.; EISENBERG, B.E. Heat stress in dairy cattle and other livestock under Southern African conditions. II Identification of areas of potential heat stress during summer by means of observes true and predicted temperature-humidity index values. Onderstepoort Journal Veterinary Research, Pretoria, SA , v.57, n.1 mar., p. 77-87,1990.

FUQUAY, J.W. Heat stress as if affects animal production. Livestock Environment, v.2, p.1133-1137, 1997.

HAHN, G.L. Management and housing of farm animals in hot environment. In: YOUSEF, M. K (Ed). Stress physiology in livestock. Cap. 2, 1985. p.151-174.

HAHN, G.L.; PARKHURRST, A.M.; GAUGHAN, J.B. Cattle respiration rate as a function of ambient temperature. Transactions of ASABE, St. Joseph, v.40, p.97-121, 1997.

HUBER, J.T. Alimentação de vacas de alta produção sob condições de estresse térmico. Bovinocultura leiteira. Piracicaba: FEALQ. 1990. p.33-48.
IGONO, M.O.; JOHNSON, H.D. Physiologic stress index of lactating dairy cows based on diurnal pattern of rectal temperature. Journal of Interdisciplinary Cycle Research,U.S. v. 21, p.303-320, 1990.

KOLB, E. Fisiologia Veterinária. 4. ed. Rio de Janeiro: Guanabara Koogan, 1987. $612 p$.

MARTELLO, L.S. Diferentes recursos de climatização e sua influência na produção de leite, na termorregulação dos animais e no investimento das instalações. Pirassununga, 2002. Dissertação (Mestrado) - Faculdade de Zootecnia e Engenharia de Alimentos. Universidade de São Paulo.

MARTELLO, L.S. Interação animalambiente: efeito do ambiente climático sobre as respostas fisiológicas e produtivas de vacas Holandesas em free-stall. Pirassununga, 2006. 113 p. Tese (Doutorado em Qualidade e Produtividade Animal) Faculdade de Zootecnia e Engenharia de Alimentos. Universidade de São Paulo

MATARAZZO, S.V. Eficiência do sistema de resfriamento adiabático evaporativo em confinamento tipo free-stall para vacas em lactação. Piracicaba, 2004. 155 p. Tese (Doutorado em Física do Ambiente Agrícola) - Escola Superior de Agricultura "Luiz de Queiroz". Universidade de São Paulo.

McDOWELL, R.E.; LEE, D.H.K.; FOHRMAN, M.H. The measurement of water and vaporation from limited areas of a normal body surface. Journal of Animal Science, Albany, NY v. 13, p. 405-416, 1954.

NÄÄS, I.A.; ARCARO JÚNIOR, I. Influência de ventilação e aspersão em sistemas de sombreamento artificial para vacas em lactação em condições de calor. Revista 
Brasileira de Engenharia Agrícola e Ambiental, Campina Grande, PB, v. 5, n. 1, p. 139-142, 2001.

PENNINGTON, J.A.; VANDEVENDER, K. Heat Stress in Dairy Cattle. Agriculture and Natural Resources. University of Arkansas, United States Department of Agriculture, and County Governments Cooperating. Disponível em :< http://www.uaex.edu> Acesso em 18/11/2005.

PERISSINOTTO, M. Avaliação da eficiência produtiva e energética de sistemas de climatização em galpões tipo freestall para confinamento de gado leiteiro. Piracicaba, 2003. 140-p. Dissertação (Mestrado em Física do Ambiente Agrícola) - Escola Superior de Agricultura "Luiz de Queiroz". Universidade de São Paulo.

REZENDE, S.O; PUGLIESI, J.B.; MELANDA, E.A.; PAULA, M.F. Mineração de dados. In: REZENDE, S.O. (Ed.). Sistemas Inteligentes: Fundamentos e Aplicações . Tamboré-Barueri-SP: Manole, 2003. Cap. 12, p. 307-335.

STOBER, M. Identificação, anamnese, regras básicas da técnica do exame clínico geral. In: ROSEMBERG (Ed). Exame clínico dos bovinos. Rio de Janeiro: Guanabara Koogan, 1993. 419-p.

STOWELL, R. R. Heat stress relief and supplemental cooling. In: DAIRY HOUSING AND EQUIPMENT SYSTEMS CONFERENCE, 2000, Ithaca. Proceedings... New York: NRAES, 2000. $\mathrm{p}-175-185$.

THOM, E.C. The discomfort index. Weatherwise, Boston, MA, v.12, n.1, p.5760, 1959. 